\title{
BMJ Open Resilience Against Depression Disparities (RADD): a protocol for a randomised comparative effectiveness trial for depression among predominantly low-income, racial/ ethnic, sexual and gender minorities
}

\begin{abstract}
Sylvanna Maria Vargas (D) , ${ }^{1,2,3}$ Ashley Wennerstrom, ${ }^{4,5}$ Nancy Alfaro, ${ }^{2}$ Thomas Belin, ${ }^{2,6}$ Krystal Griffith, ${ }^{2}$ Catherine Haywood, ${ }^{7,8}$ Felica Jones, ${ }^{9}$ Mitchell R Lunn, ${ }^{10,11}$ Diana Meyers, ${ }^{12}$ Jeanne Miranda, ${ }^{2,13}$ Juno Obedin-Maliver, ${ }^{10,11}$ Miranda Pollock, ${ }^{14}$ Cathy D Sherbourne, ${ }^{15}$ Benjamin F Springgate, ${ }^{14}$ Olivia K Sugarman, ${ }^{14,16,17}$ Emily Rey, ${ }^{18,19}$ Clarence Williams, ${ }^{9}$ Pluscedia Williams, ${ }^{9,20}$ Bowen Chung ${ }^{2,15,21,22}$
\end{abstract}

To cite: Vargas SM, Wennerstrom A, Alfaro N, et al. Resilience Against Depression Disparities (RADD): a protocol for a randomised comparative effectiveness trial for depression among predominantly lowincome, racial/ethnic, sexual and gender minorities. BMJ Open 2019;9:e031099. doi:10.1136/ bmjopen-2019-031099

\section{- Prepublication history for} this paper is available online. To view these files, please visit the journal online (http://dx.doi. org/10.1136/bmjopen-2019031099).

Received 17 April 2019 Revised 03 September 2019 Accepted 13 September 2019

Check for updates

(c) Author(s) (or their employer(s)) 2019. Re-use permitted under CC BY-NC. No commercial re-use. See rights and permissions. Published by BMJ.

For numbered affiliations see end of article.

Correspondence to Sylvanna Maria Vargas; sylvannv@usc.edu

\section{ABSTRACT}

Introduction Depression is the leading cause of adult disability and common among sexual and gender minority (SGM) adults. The current study builds on findings showing the effectiveness of depression quality improvement (QI) and delivery of cognitive behavioural therapy (CBT) skills provided by community health workers in reducing depression. Depression QI approaches across healthcare and social/community services in safety-net settings have shown improvements in mental wellness, mental health quality of life and depression over 12 months. Further, a randomised study showed improved depression among low-income racial/ethnic minorities enrolled in a CBT-informed resiliency class (Building Resilience and Increasing Community Hope (B-RICH)). The current protocol describes a comparativeness effectiveness study to evaluate whether predominantly low-income, SGM racial/ethnic minority adults randomised to a CBTinformed resiliency class have improvements in depressive symptoms over and above community-engaged QI resources and training only.

Methods and analysis The study approached three clusters of four to five programs serving predominantly SGM and racial/ethnic minority communities in the USA: two clusters in Los Angeles, California, and one in New Orleans, Louisiana. Clusters are comprised of one primary care, one mental health and two to three community agencies (eg, faith-based, social services/support, advocacy). All programs received depression QI training. The current study employed a community-partnered participatory research model to adapt the CBT-informed resiliency class, B-RICH+, to SGM communities. Study participants were screened and recruited in person from participating programs, and will complete baseline, 6 - and 12-month survey follow-ups. Participants were depressed adults (8-item Patient Health Questionnaire $\geq 10 ; \geq 18$

\section{Strengths and limitations of this study}

- This study is among the first to examine a model to reduce depression disparities and meet treatment needs among predominantly low-income, racial/ ethnic, sexual and gender minorities, an understudied population.

- We did not gather service use data from healthcare agencies but rely instead on self-report data of such use.

- Per our community-partnered discussions, we did not include any identity-based exclusion criteria, which allowed us to recruit participants who identified their sexual, gender and racial/ethnic minority status across a broad spectrum.

years of age) who provided contact information. Enrolled participants were individually randomised to $\mathrm{B}-\mathrm{RICH}+$ or depression $\mathrm{Ql}$ alone. Primary outcomes are depressive symptoms; secondary outcomes are mental health quality of life, mental wellness and physical health quality of life. Data collection for this study is ongoing.

Ethics and dissemination The current study was approved by the UCLA Institutional Review Board. Study findings will be disseminated through scientific publications and community conferences.

Trial registration number https://clinicaltrials.gov/ct2/ show/NCT02986126 


\section{INTRODUCTION}

Depressive symptoms and disorders are among the most common health conditions with a lifetime prevalence of $15 \%$ to $20 \%$ in adults. ${ }^{1}$ Depression is a leading cause of morbidity and is associated with increased healthcare costs. ${ }^{2}{ }^{3}$ In the USA, depressive symptoms have similar prevalence across racial/ethnic groups, with slightly lower rates among African Americans than Euro-Americans, ${ }^{4-8}$ however, symptoms may be more severe among depressed African Americans. ${ }^{7}$ Sexual and gender minority (SGM) adults have higher rates of depressive symptoms and disorder than those who identify as heterosexual or cisgender. ${ }^{9-14}$ A meta-analysis of research spanning four decades showed that sexual minority (SM) adults were two times as likely as heterosexual people to experience lifetime or past-year depression. ${ }^{15}$ Existing evidence suggests transgender persons experience higher rates of depression than the general cisgender population. ${ }^{9}$ Eliminating health disparities by race/ethnicity, sexual orientation and gender identity are national priorities in the USA. $^{9} 1617$

Few studies have examined depression rates among racial/ethnic minority SGM people, with some, but not all, suggesting greater rates of depression than racial/ ethnic minority heterosexuals. ${ }^{9}$ For example, one nationally representative study in the USA found higher rates of depression among Asian and Latinx SM respondents, compared with those who were heterosexual. ${ }^{11}$ In contrast, a study of SM adults in New York City showed higher rates of psychiatric disorders among Latinx and Euro-American adults in comparison to African Americans. ${ }^{18}$ Heterogeneity of depression prevalence rates among racial/ethnic and sexual minority adults may be due to variations in how sexual minority status is ascertained (eg, sexual behaviour or self-reported identity). ${ }^{9}$ Little is known about the depression of racial/ethnic minority transgender people, although a few studies suggest rates may be increased in comparison to cisgender racial/ethnic minorities. ${ }^{19}{ }^{20}$ More research is needed to understand how depression affects racial/ethnic and sexual and gender minorities.

Even less is known about the depression care or outcomes of SGM racial/ethnic minority adults. ${ }^{9}$ Lesbian, gay, bisexual, transgender and queer (LGBTQ) adults are more likely than heterosexual or cisgender persons to seek mental health services. ${ }^{9}$ A few studies have found similar patterns among racial/ethnic minority SMs in comparison to heterosexuals, ${ }^{21}$ and some differences between SM racial/ethnic minorities and Euro-Americans. ${ }^{22}$ However, many adults with depression only receive treatment in primary care, where depression is common. ${ }^{2323}$ Yet, in primary care, up to $50 \%$ of adult patients with depression may not be identified. ${ }^{24}$ Of those whose depression is identified, half, particularly racial/ ethnic minorities, may not receive guideline-concordant treatment. ${ }^{1625} 26$ Racial/ethnic minority patients receive lower quality care and show worse outcomes for depression than Euro-Americans. ${ }^{827-30}$ While limited research has examined quality of care received by SGM people, some studies show that perceived quality and utilisation of care is affected by providers' knowledge about LGBTQ issues. $^{9}$

To improve quality in depression treatment delivery, numerous studies have developed collaborative care models that integrate mental health treatment within primary care settings. ${ }^{31}{ }^{32}$ As part of this effort, quality improvement (QI) programmes provide training on evidence-based interventions to healthcare providers. ${ }^{32-36}$ QI programmes are traditionally developed and disseminated by healthcare experts as part of primary care integration efforts to train providers in mental health practices. ${ }^{34-36}$ In over 79 randomised controlled trials, depression QI programmes reduced depression among diverse settings and patients. ${ }^{32} 33$ Even though QI programmes may reduce racial/ethnic outcome disparities in adults, residual symptoms and quality of life limitations may persist over time. ${ }^{37} 38$ Furthermore, limited availability of primary care limits the implementation of evidencebased, depression QI programmes in under-resourced communities. ${ }^{16} 3239$ These problems are exacerbated by an array of factors contributing to mental health disparities among low-income SGM and racial/ethnic minority communities, including stigma, variable service availability, quality and coordination,, $16263140-42$ and distrust in healthcare services and research due to the legacy of research abuses and unequal treatment. ${ }^{43-45}$

In general, limited evidence exists on the effect of depression interventions on outcomes for HIV-negative SGM people, particularly those who are also racial/ ethnic minorities in low-resourced communities. ${ }^{9} 13144647$ However, structured psychotherapies — such as cognitive behavioural therapy $(\mathrm{CBT})^{48}$ - are evidence-based treatments for improving depression outcomes and are effective among low-income, racial/ethnic minorities. ${ }^{34-36} 4950$ A recent randomised-controlled trial similarly showed the effect of community and academic partnerships through developing a 7-session community health worker-led psychoeducation class (Building Resilience and Increasing Community Hope (B-RICH)), which was more effective than case management alone in reducing depression among racial/ethnic minorities (Chung et al, B-RICH). Similarly, prior work that extends depression QI training and implementation support to healthcare and community-based organisations (eg, faith-based, social services, senior centres) demonstrated that planning together with such agencies improves outcomes such as mental health-related quality of life and mental well-being over and above technical support alone in racial/ethnic low-income communities up to 3 years. ${ }^{34-365152}$ This work points to the important role of working with patients and trusted community organisations to determine how and where to provide access to mental healthcare with trusted community organisations. Although a number of evidence-based approaches have been shown to improve depression outcomes more generally, few studies have focused on SGM racial/ethnic minorities. 


\section{Current study}

Building on over 10 years of collaborative work in addressing mental health in Louisiana and California in the USA, our team employed a community-partnered participatory research (CPPR) approach ${ }^{53}$ to develop the SGM-focused Resilience Against Depression Disparities (RADD) study in New Orleans and Los Angeles. Using CPPR principles such as equal power, two-way knowledge exchange and shared resources, we engaged a wide variety of community and academic partners to shape the design, framing, scope, research questions, proposed measures and materials for this study.

As we created our study, partners emphasised that low-income, SGM racial/ethnic minorities likely faced higher rates of depression due to stigma, social exclusion, increased risk for interpersonal violence and added burden in seeking depression care. They also explained that in racial/ethnic minority, under-resourced communities, many SGM adults approach 'coming out' or disclosure of SGM status to non-trusted organisations with caution, since this might increase SGM patients' risk of discrimination. This therefore signalled the importance of conducting depression treatment within trusted spaces and organisations. Further, as described below, our research and intervention development were sensitive to potential adverse consequences of unintended disclosure of SGM status.

Given the substantial evidence in the literature about approaches to improve depression outcomes even for racial/ethnic minorities, our partners felt that any randomised studies with a 'usual care' or waitlist condition for depression would be unethical despite the limited information about treatments for SGM racial/ethnic minorities. Finally, our community partners indicated that resilience-focused - rather than disease-focused - interventions may help address existing barriers to care. Therefore, the current study developed B-RICH+, a resiliency-oriented, CBT psychoeducational intervention tailored to SGM individuals. Based on our discussions with study partners, we developed the following objective: to evaluate the added value of SGM-tailored B-RICH+ overand above SGM-tailored depression QI resources and training on improving adult patients' depressive symptoms and mental health quality of life, mental wellness and physical health quality of life.

\section{METHOD}

\section{Study design}

RADD is an individual-level, randomised comparative effectiveness study. Our trial assesses the effects of two interventions on depressive symptoms and participant engagement in service use, including speciality mental health services, primary care, substance abuse treatment programme, social services agencies and community-based and faith-based services where participants report receiving support. The two interventions compare: (1) B-RICH+, a depression CBT-based psychoeducation group; and (2) Resource for Services (RS), an evidencebased, program-level, SGM-tailored depression QI providing resources and training to enrolled healthcare and community agencies. All organisations from which participants were sampled were provided the opportunity to receive RS, with half of individual participants randomised to receive B-RICH+. Our study is registered under ClinicalTrials.gov (https://clinicaltrials.gov/ct2/ show/NCT02986126) and will take place from August 2016 to March 2020.

Figure 1 provides an overview of the study. Participants were screened in person at community agencies and clinics, and then called on the telephone to complete baseline, 6- and 12-month follow-up surveys. Telephone surveys during baseline and follow-ups will be collected by the RAND Survey Research Group. Randomisation was determined based on a combination of demographics, site location and language during baseline interview. Participants were informed about their randomisation arm over the telephone at the end of their baseline interview. Participants who were randomised to B-RICH+ were contacted by the community health worker leading the resiliency class. To the extent possible, classes were held at times and locations convenient for the participants. Participants were informed that their participation in the RADD study was not contingent on their participating in the classes.

\section{Patient and public involvement}

The RADD study employs a CPPR model. Community advocates, researchers, patients, providers and other stakeholders are collaborating throughout all phases of the research process ${ }^{53-56}$ including development of the research question, adapting the study and intervention materials to SGM communities and ongoing discussions about data collection and reducing participant burden, study data analysis and dissemination of findings. Our community partners belonged to the same diverse communities as did our study participants. Our community partners explained that although New Orleans and Los Angeles may be more SGM-friendly than other cities in the USA more generally, acceptance of SGM did not extend to specific neighbourhoods, where some people may fear 'coming out'. Therefore, per community partners' recommendations, we avoided unintended disclosure of our participants' SGM status by using some study materials that did not indicate the SGM focus. For example, our study name focused on depression disparities but did not describe the population to avoid unintentionally outing a participant over the phone. Similarly, our contact materials (eg, postcards, letters, emails) did not reference SGM groups. However, community partners also stressed the importance of affirming sexual orientation and gender identity. Therefore, we included rainbow themes throughout our study materials (eg, pamphlets, handouts) to make them SGM-friendly and affirming.

The research is being conducted by the Community and Patient Powered Research Network (CPPRN) in 


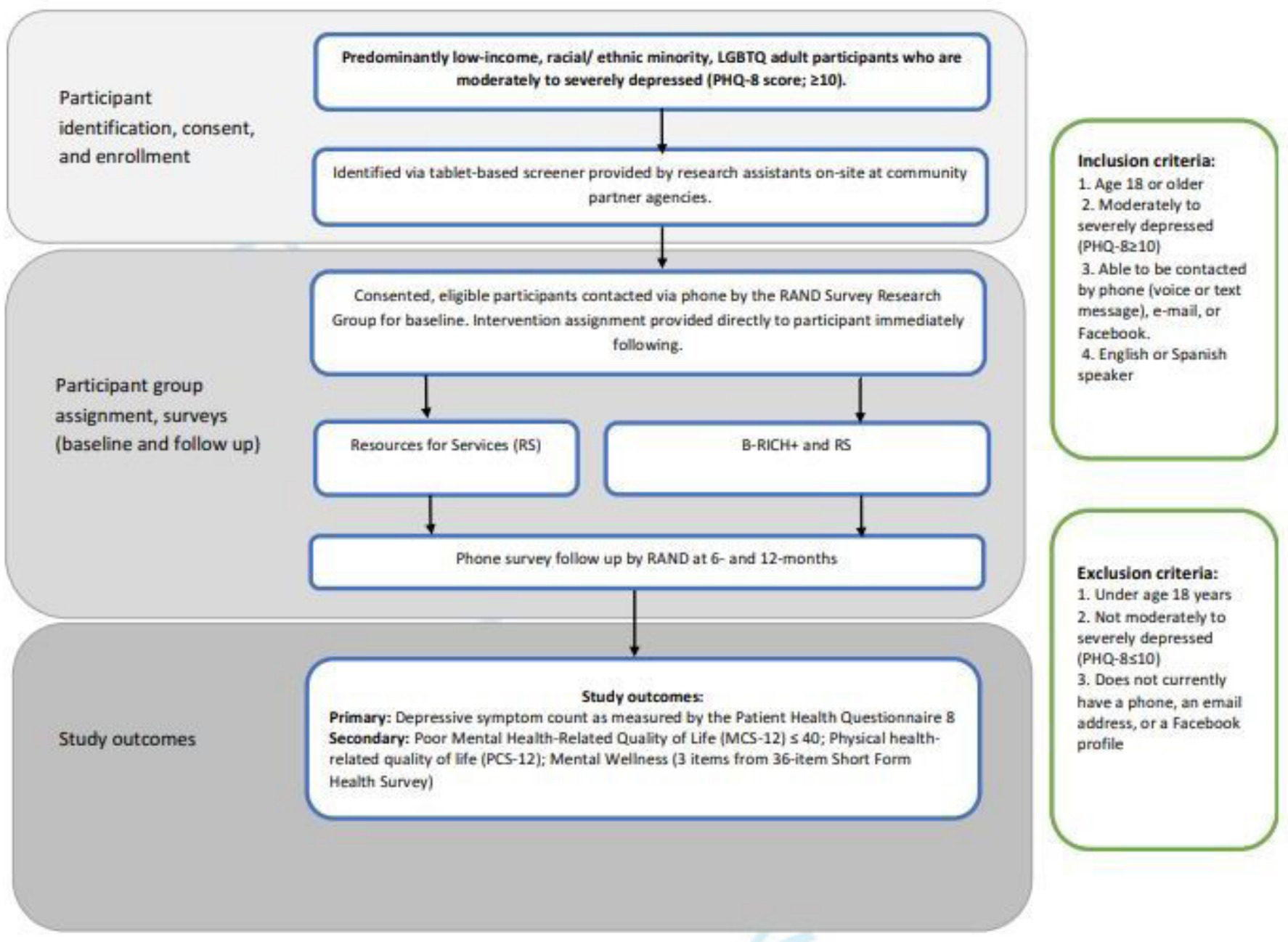

Figure 1 Study overview illustrating recruitment, study enrolment and randomisation, data collection waves and study outcomes. B-RICH+, Building Resilience and Increasing Community Hope+; LGBTQ, lesbian, gay, bisexual, transgender and queer; PHQ-8, 8-item Patient Health Questionnaire.

Los Angeles and New Orleans. The CPPRN is part of the Patient-Centered Outcomes Research Institute's network of Patient-Powered Research Network and Clinical Data Research Networks collectively known as PCORnet.

\section{Interventions}

\section{Cultural adaptation process}

We adapted both study interventions through meetings with individuals who represented the target community. We maintained the active core ingredients in depression treatments and developed relevant examples and changes to make the treatments more relevant to the community. ${ }^{57}$ Specific adaptations are provided below under each intervention description.

$R S$ is an evidence-based depression QI toolkit developed for primary care ${ }^{5658}$ but adapted for health- and community-based programme clusters ${ }^{36} 477^{59-61}$ with tailoring for SGM individuals. RS provides support training to staff in participating agencies: licensed providers in clinical assessment, medication management and CBT; all staff in team management and non-clinical staff $(e g$, case managers) in addressing patient safety, screening, behavioural management skills (eg, behavioural activation, brief problem-solving) to enable education coordination and referral for depressed patients. As part of our cultural adaptation process, we added a training on SGM sensitivity, including education about sexual orientation and gender identity and issues that SGM persons face in seeking healthcare and other services. RS is offered as 12 webinars (three each on team management, medication management, psychotherapy and case management) with primary care on-site training. ${ }^{34-36}$

$B$-RICH+ is a 7 -session psychoeducational intervention delivered by community health workers who belong to the SGM racial/ethnic minority community. The class is a manualised, CBT-informed intervention that teaches people skills to improve their mood and address depression symptoms. Community partners identified priorities for coping support and resources, and they adapted content from an existing CBT-based manual to support resiliency. We adapted examples and issues that were 
relevant to SGM persons, such as finding affirming social support and cognitive restructuring around how to cope with discrimination. Each session is 90 to $120 \mathrm{~min}$, and classes can be delivered in both English and Spanish. This class does not provide therapy, but rather is designed to build skills for improving mood and enhancing resiliency in the face of stress. Classes are supplemented with optional automated mobile text reminders about basic concepts and follow-up for care (eg, appointment reminders). Text messages are delivered by an electronic platform known as CHORUS, ${ }^{62}$ the content of which was created through a series of participatory workshops with community stakeholders and designed to provide basic and quick tips for coping support and resources. Community partners identified priorities for coping support and resources, and they adapted content from an existing CBT-based manual to support resiliency.

\section{Participants}

We enrolled a randomised sample comprised of Englishspeaking and Spanish-speaking adults ( $\geq 18$ years $)$ who screened positive for depression on an 8-item Patient Health Questionnaire $((\mathrm{PHQ}-8) \geq 10) .{ }^{63}$ Given that prior research and our community partners mentioned that SGM racial/ethnic minorities may not identify as LGBTQ ${ }^{9}$ we did not create any inclusion/exclusion criteria around self-reported identity. Instead, we opted to recruit participants from organisations in Los Angeles and New Orleans that serve high proportions of SGM individuals, including federally qualified health centres (primary care, mental health and substance abuse centres), community-based agencies (faith-based, homeless-serving and those providing social services) and other community-trusted locations (LGBTQ-friendly bars, gyms). Of 1825 people screened, 465 met study eligibility criteria, 385 enrolled and 256 completed a set of baseline measures. We are conducting 6 month (estimated $\mathrm{n}=242$, based on rate of attrition from past studies) and 12 month (estimated $\mathrm{n}=224$ ) follow-ups via telephone. Participants who did not speak English or Spanish or were too impaired to complete screening were excluded.

\section{Informed consent}

Persons who agreed to be screened for eligibility were provided informed consent via an electronic platform, CHORUS, which allows for capture of an electronic signature on an electronic tablet. Participants were given a paper copy of the consent form. Those who met the eligibility criteria after completing the PHQ-8 screener were invited to enrol in the study. Enrolment included signing an additional electronic consent form that gave permission for survey staff to call at baseline, 6 and 12 month follow-up. Enrolment entailed providing contact information so that participants can be contacted later for baseline and follow-up surveys. Participants were informed that they can refuse to participate in any study component and may leave questions unanswered and remain in the study, with no consequences. To protect confidentiality, the current study obtained a Certificate of Confidentiality from the National Institute of Health, allowing the study to refuse to disclose research information to any civil, criminal, administrative, legislative or other preceding, except as required by law.

\section{Data sources}

Patient data are obtained from the study screener completed in person at service sites, as well as baseline, 6 and 12 month follow-up telephone survey interviews that are independent of visits for services. All participant data will be captured on secure computer systems. All data will be de-identified by assigning participants an identification number in order to protect confidentiality.

\section{Primary outcome}

Our primary outcome is change in depressive symptoms as measured by the PHQ- $8{ }^{64}$ score at 6 - and 12 -months after baseline survey completion and randomisation.

\section{Secondary outcomes}

Secondary outcomes measured at 6 and 12 months post-intervention include mental health-related quality of life, as measured by the mental composite score (MCS) $\leq 40$ from the 12-itemshort-form health survey $(\mathrm{SF}-12)^{6465}$, a 3-item measure of mental wellness from the 36-itemshort-form health survey ${ }^{667}$ (eg, feeling calm or peaceful, having energy and being happy over the last 4 weeks) and physical health quality of life using the 12-item physical composite score from the SF-12. ${ }^{64} 65$

\section{Additional study measures}

Telephone baseline and follow-up survey data include depression, anxiety and bipolar disorder status assessed using the Computerised Adaptive Test-Mental Health (CAT-MH) tool. ${ }^{68}$ CAT-MH produces continuous symptom severity scores in the past 2 weeks by adapting the number of survey items based on participant response. Participants endorsing suicidality during this survey will be immediately contacted by a study clinician on call. Once a participant is identified by the CAT-MH of being at high risk for suicide, an automatic message will be automated to ultimately page a study clinician, and the participant's survey will continue over the phone. The study clinician will contact the survey centre to receive the participant's information. The study clinician will then call the participant within $30 \mathrm{~min}$ of being paged, once the participant has ended the phone survey. The clinician will conduct a risk assessment and provide a referral to care from a list of clinics in Los Angeles and New Orleans and the phone number for the US National Suicide Hotline. In cases of imminent danger, the study clinician will advise the participant to visit the nearest emergency room or the provider may call 911. Similarly, if a participant reports homocidality or cases of child or elderly abuse, a study clinician will be notified and contact the participant to conduct a risk assessment and make any necessary reporting as required by law. 
Phone surveys also include indicators of history of hospitalisation and service use ${ }^{34}{ }^{35}$ medication, ${ }^{69}$ sexual behaviour, ${ }^{70}$ HIV testing status, ${ }^{71}$ post-traumatic stress, ${ }^{72}$ alcohol problems ${ }^{73}$ substance abuse ${ }^{74}$ chronic physical conditions, ${ }^{65}$ life difficulties ${ }^{42}$ resilience, ${ }^{75}$ social support, ${ }^{76}$ employment status, work missed, perceived discrimination, ${ }^{77}$ internalised homophobia ${ }^{78}{ }^{79}$ and 'outness' (ie, level of comfort and disclosure to others regarding sexual orientation and gender identity). ${ }^{80}$ All study measures are summarised in table 1 . Measures were selected and developed in conjunction with community partners, who advised many of the topics assessed (eg, discrimination, outness, internalised homophobia) are important stressors relevant to depression in the community.

\section{Data safety and monitoring}

A Data Safety and Monitoring Board (DSMB) comprised of a member of the community, a statistician and a behavioural health services researcher was formed, to provide external, independent oversight of the study. As an external, independent oversight board, DSMB members will monitor the study feasibility, data integrity and safety and ensure that monitoring is timely and effective. The DSMB will meet every other month on 1 hour conference calls and webinars from month 6 through month 30 of the proposed demonstration. All members of the DSMB have no conflicts of interests and are independent from the study funding agency.

\section{Statistical analysis}

We will use an intent-to-treat analysis in two steps. First, we will examine intervention effect at 6 months, comparing outcomes for B-RICH+ and RS versus RS only. We will fit linear regression models for continuous variables (PHQ-8) with intervention status as the independent variable, logistic regression models for binary outcomes (secondary outcome: MCS-12 $\leq 40$ ) and Poisson (or negative binomial) for number of service use visits using 6 and 12 month data separately controlling for patient baseline measures for the same outcome, area (Los Angeles or New Orleans) and type of service use (health or community programme). Second, we will examine B-RICH+ effects relative to RS over 12 months. In models which include all time periods, we will account for repeated measurements within patients. We will treat time as a categorical variable and examine fixed effects for time, intervention condition and their interaction with regression adjustment for baseline and covariates. These models will allow us to examine the intervention effects at each time point, to evaluate effectiveness and sustainability, to measure the difference in effects between two follow-ups and to calculate average effects over time. We will use SAS MIXED for linear regressions, GLIMMIX for logistic regressions and Poisson regressions for repeated measures. ${ }^{81}$ We will perform sensitivity analyses with time as a continuous variable (ie, data collection time points) in mixed models.

\section{Missing data}

The main sources of missing data are item-level or participant-level non-response. By using telephone surveys assisted by computer programming to collect survey data, we minimise item non-response. We will do logical imputation for items and a hot-deck multiple imputation technique using a predictive mean matching method for item non-response, ${ }^{82} 83$ combining results from multiply imputed data using SAS PROC MIANALYZE to account for uncertainty in imputed values. ${ }^{83}$ Multiple logistic regressions will be used to evaluate baseline characteristics of participants that may differentiate dropouts from completers. To assess the extent to which attrition could bias results, we will employ techniques such as pattern mixture models as alternatives to imputation ${ }^{84-87}$ under ignorable models (ie, assuming items are missed randomly) ${ }^{83889}$ In prior work, we incorporated weighting adjustments to represent units in the population that are not enrolled, ${ }^{90}$ and have used an approximate Bayesian bootstrap, ${ }^{91}$ stratifying on propensity scores that capture probability of response, to represent units in the sample where data are not available at all time points. Overall analyses will reflect weighting and multiple imputation for unit non-response. We will conduct sensitivity analyses reflecting uncertainty associated with predictions within models and in the choice of models, and will report sources of missing data at item-unit and participant-unit levels in reports and discuss potential impact of missing data on interpretation of findings.

\section{Weights}

We will create weights to account for the sampling design and to control for potential non-response bias. ${ }^{92}$ For example, for the 6 month follow-up survey, we will construct attrition weights by fitting logistic regression models to predict follow-up status from baseline predictors including socio-demographic and clinical variables stratified by the two intervention arms. The fitted logistic regression models will be used to derive the predicted probability for each individual respondent to remain in follow-up. The reciprocal of the predicted probability will be then used as the 6 month attrition weight for each participant. Similar methods will be used for 12 month analyses.

\section{Multiple comparisons}

We will consider Bonferroni and related methods incorporating bounds on the probability of a single false finding of significance. We will consider the false discovery rate comparing observed significance with expected order statistics from a uniform distribution.

\section{DISCUSSION}

Little is known about how to address the depression that low-income SGM racial/ethnic-minorities face. Our study is among the first to use an randomised controlled trial to test whether a community health worker-led 
Table 1 Overview of study measures

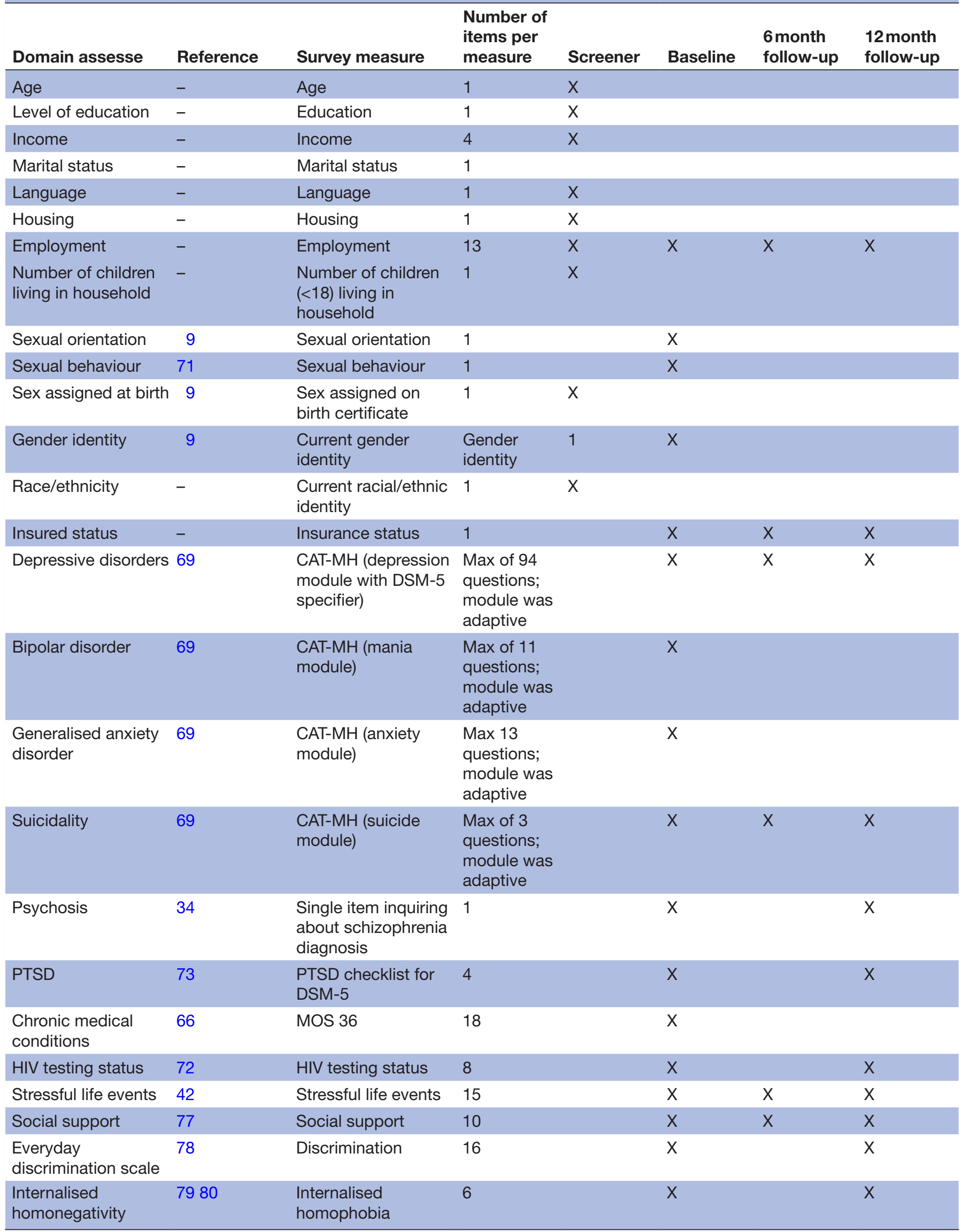


Table 1 Continued

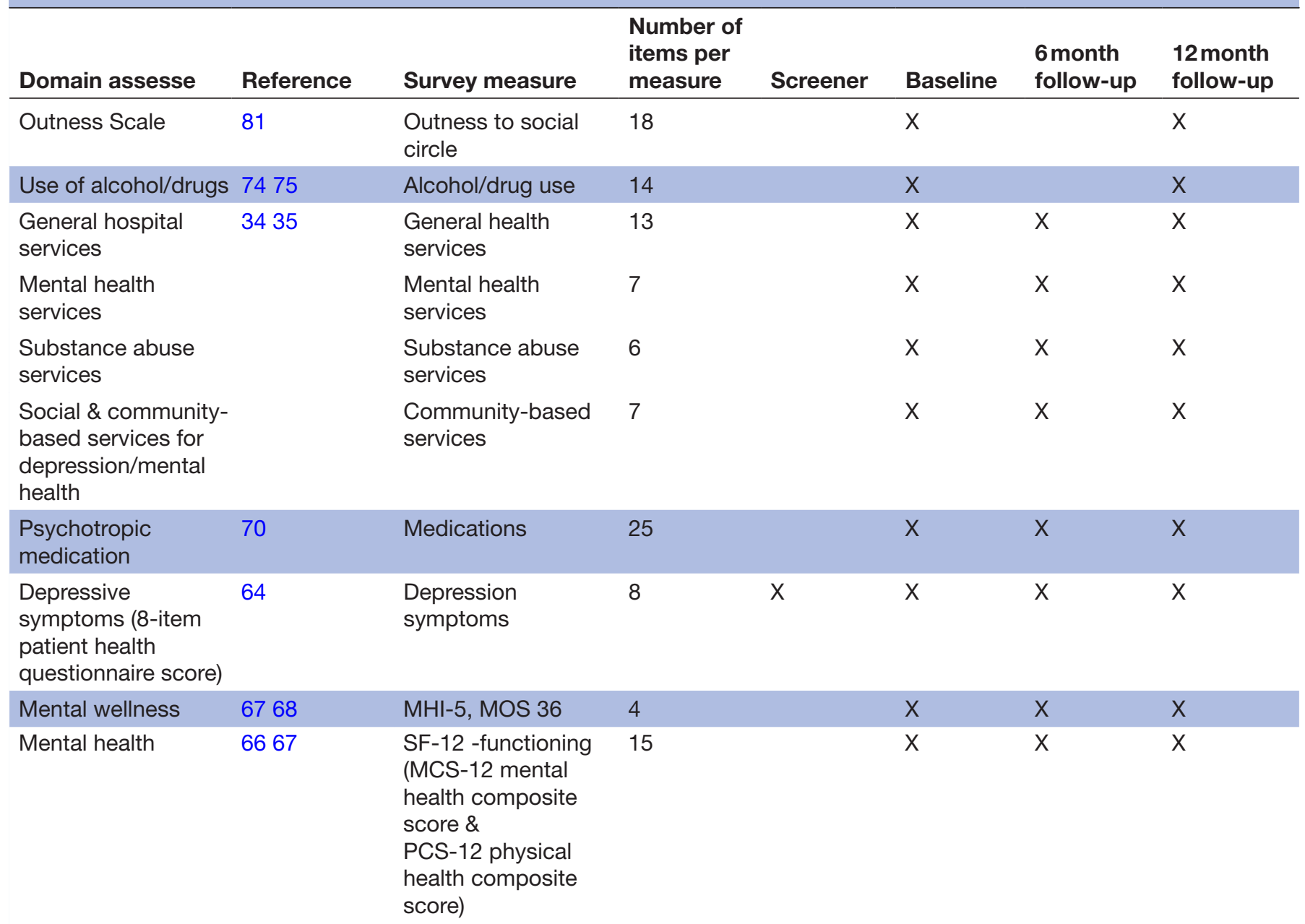

CAT-MH, Computerised Adaptive Test-Mental Health; MCS, mental composite score; MHI, Mental Health Inventory; MOS, Medical Outcomes Study; PCS, physical composite score; PTSD, post-traumatic stress disorder; SF-12, 12-item short-form health survey.

psychoeducational, resilience-focused intervention can improve depression among sexual, gender and racial/ ethnic minorities in comparison to a culturally-adapted QI training. The study has several areas of potential significance. First, this study will provide important data to address a key scientific gap on how to improve depression outcomes for predominantly racial/ethnic SGM adults, especially for HIV-negative SGM groups. These data may inform how medical homes, community-based agencies and social service providers can implement evidence-based depression QI approaches and psychoeducation delivered by community health workers. Furthermore, this study may provide insight into how healthcare providers can best address mental health quality and outcome disparities with patient and community input. Ultimately, patient and stakeholder engagement through partnered research may accelerate science and translation of findings into practice.

\section{Limitations}

Although Los Angeles and New Orleans are comparatively SGM-friendly cities, this is not true for all their communities. Given that differences exist in how SGM racial/ethnic minorities identify, we targeted recruitment from sites that were considered relatively 'safe-spaces' for SGM persons. Our study likely includes a subset of SGM racial/ethnic minorities, who do not necessarily generalise to other communities. Given the long history of community-partnered research in our team, it is possible our study findings may not generalise to other communities without similar partnerships. Furthermore, our study relies on self-report measures, which are subjective. For example, we asked participants about their service utilisation, but did not rely on chart or claims data from healthcare agencies. However, prior studies have shown that self-reported health service utilisation is valid. ${ }^{9394}$

\section{Ethics and dissemination}

Louisiana State University and RAND have deferred approval to UCLA's IRB. Analysis of study data, scientific presentations and peer-reviewed manuscripts will all be done in partnership with community and client partners. In addition to scientific publications, as part of our project grant requirements, we will promote dissemination to 
stakeholders through community conferences, which will take place in Los Angeles and New Orleans throughout the study. We will convene a partnered dissemination workgroup to develop strategies to disseminate research study findings to the local communities. These dissemination efforts may include online materials, email distribution lists, church meetings, printed storybooks, mailings to networks of community partners, presentations in community venues (eg, movie theatres, neighbourhood association meetings, booths at community fairs), social media and/or special features in newspapers/on radio.

\section{Author affiliations}

${ }^{1}$ Psychology, University of Southern California, Los Angeles, California, USA

${ }^{2}$ Psychiatry and Biobehavioral Sciences, Semel Institute for Neuroscience and Human Behavior, UCLA, Los Angeles, California, USA

${ }^{3}$ Preventive Medicine, University of Southern California, Los Angeles, California, United States

${ }^{4}$ School of Medicine, Tulane University, New Orleans, Louisiana, USA

${ }^{5}$ LSU Health Sciences Center New Orleans, Louisiana State University, New Orleans, Louisiana, United States

${ }^{6}$ Department of Biostatistics, Fielding School of Public Health, UCLA, Los Angeles, California, USA

${ }^{7}$ Tulane Prevention Research Center, Tulane University, New Orleans, Louisiana, USA

${ }^{8}$ Women with a Vision, Inc, New Orleans, Louisiana, USA

${ }^{9}$ Healthy African American Families II, Los Angeles, California, USA

${ }^{10}$ Department of Medicine, Stanford University School of Medicine, Stanford, California, USA

${ }^{11}$ The PRIDE Study, Stanford University School of Medicine, Stanford, California, United States

${ }^{12}$ Saint Anna's Episcopal Church, New Orleans, Louisiana, USA

${ }^{13}$ Health Policy and Management, Fielding School of Public Health, UCLA, Los Angeles, California, United States

${ }^{14}$ Section of Community and Population Medicine, Department of Medicine,

Louisiana State University School of Medicine, New Orleans, Louisiana, USA

${ }^{15}$ RAND Corporation, Los Angeles, California, USA

${ }^{16}$ Center for Health Care Value and Equity, LSU Health Sciences Center, New Orleans, Louisiana, United States

${ }^{17}$ School of Public Health, LSU Health Sciences Center, New Orleans, California, United States

${ }^{18}$ LGBT Community Center, New Orleans, Louisiana, USA

${ }^{19}$ Let's Hatch Media, New Orleans, Louisiana, USA

${ }^{20}$ Department of Preventive and Social Medicine, Charles R Drew University of Medicine and Science, Los Angeles, California, USA

${ }^{21}$ Department of Psychiatry, Harbor-UCLA Medical Center, Torrance, California, United States

${ }^{22}$ Department of Mental Health, County of Los Angeles, Los Angeles, California, United States

\section{Twitter Juno Obedin-Maliver @ObedinMaliver}

Contributors Contributorship: All authors listed below (1) made substantial contributions to the design of the study, collection of data and/or analyses of the data, (2) drafted or critically revised this manuscript for important intellectual content, (3) reviewed and approved the final draft before submission and (4) agreed to be accountable for all aspects of the study and manuscript. Following CPPR model of shared power, authors for this manuscript include academic researchers and community advisors. Specific contributions for points (1) and (2) are listed below: SMV: (1) design of the study and analyses of data, (2) drafted and critically revised manuscript; AW: (1) design of the study and analyses of data, (2) drafted and critically revised manuscript; NA: (1) design of the study and collection of data, (2) critically revised manuscript; TB: (1) analyses of data, (2) critically revised manuscript; KG: (1) design of the study and collection of data, (2) critically revised manuscript; CH: (1) design of the study, (2) critically revised manuscript; FJ: (1) design of the study, (2) critically revised manuscript; MRL: (1) design of the study, (2) critically revised manuscript; DM: (1) design of the study, (2) critically revised manuscript; JM: (1) design of the study, (2) critically revised manuscript; JOM: (1) design of the study, (2) critically revised manuscript; MP: (1) design of the study, collection of data and analyses of data, (2) critically revised manuscript; CDS: (1) design of the study, (2) critically revised manuscript; BFS: (1) design of the study, (2) critically revised manuscript; OKS: (1) design of the study, (2) critically revised manuscript; ER: (1) analyses of data, (2) critically revised manuscript; CW: (1) design of the study, (2) critically revised manuscript; PW: (1) design of the study, (2) critically revised manuscript; $\mathrm{BC}$ : (1) design of the study and analyses of data, (2) drafted and critically revised manuscript, (3) reviewed and approved the final draft before submission, (4) agreed to be accountable for all aspects of the study and the manuscript.

Funding This study was supported by the Patient-Centered Outcomes Research Institute (PCORI, www.pcori.org), grant number: PPRND-1507-32173. SMV was supported through a Ford Foundation Predoctoral Fellowship. MRL and JOM were partially supported through a PCORI Award (PPRN-1501-26848) to MRL. JOM was also partially supported by a grant from the National Institute of Diabetes, Digestive and Kidney Disorders (1K12DK111028). JM was partly supported through a Substance Abuse and Mental Health Services Administration grant (SM080041-01) and a National Institute of Mental Health grant (P30MH058107-21). BC was also supported by the UCLA Clinical and Translational Science Institute/ National Center for Advancing Translational Sciences grant (UL1TR001881). The statements in this article are solely the responsibility of the authors and do not necessarily represent the views of PCORI, its Board of Governors or Methodology Committee.

Competing interests None declared.

Patient consent for publication Not required.

Ethics approval The current study was approved by the UCLA Institutional Review Board.

Provenance and peer review Not commissioned; externally peer reviewed.

Open access This is an open access article distributed in accordance with the Creative Commons Attribution Non Commercial (CC BY-NC 4.0) license, which permits others to distribute, remix, adapt, build upon this work non-commercially, and license their derivative works on different terms, provided the original work is properly cited, appropriate credit is given, any changes made indicated, and the use is non-commercial. See: http://creativecommons.org/licenses/by-nc/4.0/.

ORCID iD

Sylvanna Maria Vargas http://orcid.org/0000-0002-6824-1842

\section{REFERENCES}

1 Kessler RC, Chiu WT, Demler O, et al. Prevalence, severity, and comorbidity of 12-month DSM-IV disorders in the National comorbidity survey replication. Arch Gen Psychiatry 2005;62:617-27.

2 Simon G, Ormel J, VonKorff M, et al. Health care costs associated with depressive and anxiety disorders in primary care. Am J Psychiatry 1995;152:352-7.

3 Unützer J, Patrick DL, Simon G, et al. Depressive symptoms and the cost of health services in $\mathrm{HMO}$ patients aged 65 years and older. A 4-year prospective study. JAMA 1997;277:1618-23.

4 Abe-Kim J, Takeuchi DT, Hong S, et al. Use of mental health-related services among immigrant and US-born Asian Americans: results from the National Latino and Asian American study. Am J Public Health 2007;97:91-8.

5 Alegria M, Takeuchi D, Canino G, et al. Considering context, place and culture: the National Latino and Asian American study. Int J Methods Psychiatr Res 2004;13:208-20.

6 Takeuchi DT, Alegría M, Jackson JS, et al. Immigration and mental health: diverse findings in Asian, black, and Latino populations. Am J Public Health 2007;97:11-12.

7 Williams DR, González HM, Neighbors H, et al. Prevalence and distribution of major depressive disorder in African Americans, Caribbean blacks, and non-Hispanic whites: results from the National survey of American life. Arch Gen Psychiatry 2007;64:305-15.

8 González HM, Vega WA, Williams DR, et al. Depression care in the United States: too little for too few. Arch Gen Psychiatry 2010;67:37-46.

9 Institute of Medicine. The Health of Lesbian, Gay, Bisexual, and Transgender People: Building a Foundation for Better Understanding. Washington, D.C.: National Academies Press, 2011.

10 Bostwick WB, Boyd CJ, Hughes TL, et al. Dimensions of sexual orientation and the prevalence of mood and anxiety disorders in the United States. Am J Public Health 2010;100:468-75.

11 Cochran SD, Mays VM, Alegria M, et al. Mental health and substance use disorders among Latino and Asian American lesbian, gay, and bisexual adults. J Consult Clin Psychol 2007;75:785-94. 
12 Operario D, Gamarel KE, Grin BM, et al. Sexual minority health disparities in adult men and women in the United States: National health and nutrition examination survey, 2001-2010. Am J Public Health 2015;105:e27-34.

13 Strutz KL, Herring AH, Halpern CT. Health disparities among young adult sexual minorities in the U.S. Am J Prev Med 2015;48:76-88.

14 Herek GM, Garnets LD. Sexual orientation and mental health. Annu Rev Clin Psychol 2007;3:353-75.

15 King M, Semlyen J, Tai SS, et al. A systematic review of mental disorder, suicide, and deliberate self harm in lesbian, gay and bisexual people. BMC Psychiatry 2008;8:70.

16 U.S. Department of Health and Human Services. Mental Health: Culture, Race, andEthnicity - A Supplement To Mental Health: $A$ Report of the Surgeon General. Rockville: USDHHS, 2001.

17 U.S. Department of Health and Human Services. Advancing LGBTQT Health \& Well-Being: 2014 Report, 2014. Available: http://www.hhs. gov/programs/topic-sites/LGBTQt/index.html [Accessed October 9, 2015]

18 Meyer IH, Dietrich J, Schwartz S. Lifetime prevalence of mental disorders and suicide attempts in diverse lesbian, gay, and bisexual populations. Am J Public Health 2008;98:1004-6.

19 Lytle MC, Blosnich JR, Kamen C. The association of multiple identities with Self-Directed violence and depression among transgender individuals. Suicide Life Threat Behav 2016;46:535-44.

20 Bauermeister JA, Goldenberg T, Connochie D, et al. Psychosocial disparities among racial/ethnic minority transgender young adults and young men who have sex with men living in Detroit. Transgend Health 2016;1:279-90.

21 Matthews AK, Hughes TL. Mental health service use by African American women: exploration of subpopulation differences. Cultur Divers Ethnic Minor Psychol 2001;7:75-87.

22 Macapagal K, Bhatia R, Greene GJ. Differences in healthcare access, use, and experiences within a community sample of racially diverse lesbian, gay, bisexual, transgender, and questioning emerging adults. LGBT Health 2016;3:434-42.

23 Unützer J, Katon W, Williams JW, et al. Improving primary care for depression in late life: the design of a multicenter randomized trial. Med Care 2001;39:785-99.

24 Mitchell AJ, Vaze A, Rao S. Clinical diagnosis of depression in primary care: a meta-analysis. Lancet 2009;374:609-19.

25 Wells KB. Caring for depression in primary care: defining and illustrating the policy context. J Clin Psychiatry 1997;58 Suppl $1: 24-7$.

26 Institute of Medicine. Unequal Treatment: Confronting Racial and Ethnic Disparities in Health Care. Washington, D.C.: National Academies Press, 2003.

27 Wang PS, Berglund P, Kessler RC. Recent care of common mental disorders in the United States. J Gen Intern Med 2000;15:284-92.

28 Miranda J, McGuire TG, Williams DR, et al. Mental health in the context of health disparities. Am J Psychiatry 2008;165:1102-8.

29 Kessler RC, Heeringa S, Lakoma MD, et al. Individual and societal effects of mental disorders on earnings in the United States: results from the National comorbidity survey replication. Am J Psychiatry 2008;165:703

30 González HM, Tarraf W, Whitfield KE, et al. The epidemiology of major depression and ethnicity in the United States. J Psychiatr Res 2010;44:1043-51.

31 SAMHSA-HRSA. Behavioral Health Homes for People with Mental Health \& Substance Use Conditions: The Core Clinical Features. Wasington: Center for Integrated Health Solutions, 2012.

32 Archer J, Bower P, Gilbody S, et al. Collaborative care for depression and anxiety problems. Cochrane Database Syst Rev 2012;10.

33 Gilbody S, Bower P, Fletcher J, et al. Collaborative care for depression: a cumulative meta-analysis and review of longer-term outcomes. Arch Intern Med 2006;166:2314.

34 Wells KB, Jones L, Chung B, et al. Community-partnered clusterrandomized comparative effectiveness trial of community engagement and planning or resources for services to address depression disparities. J Gen Intern Med 2013;28:1268-78.

35 Chung B, Ong M, Ettner SL, et al. 12-Month outcomes of community engagement versus technical assistance to implement depression collaborative care: a partnered, cluster, randomized, comparative effectiveness trial. Ann Intern Med 2014;161:S23-34.

36 Chung B, Ngo VK, Ong MK, et al. Participation in training for depression care quality improvement: a randomized trial of community engagement or technical support. Psychiatr Serv 2015;66:831-9.

37 Plough A, Fielding JE, Chandra A, et al. Building community disaster resilience: perspectives from a large urban County department of public health. Am J Public Health 2013;103:1190-7.
38 Areán PA, Ayalon L, Hunkeler E, et al. Improving depression care for older, minority patients in primary care. Med Care 2005:43:381-90.

39 U.S. Department of Health and Human Services. Hhs action plan to reduce racial and ethnic disparities: a nation free of disparities in health and health care. Washington, D.C: U.S. Department of Health and Human Services, 2011

40 U.S. Department of Health and Human Services. New Freedom Commission on MentalHealth: Achieving the Promise: Transforming Mental Health in America. Final report. Rockville: USDHHS: SMA03-3832, 2003

41 Institute of Medicine. Adaptation to Mental Health and Addictive Disorders: Improving the Quality of Health Care for Mental and Substance-Use Conditions. Washington, D.C: National Academies Press, 2006.

42 Roeloffs C, Sherbourne C, Unützer J, et al. Stigma and depression among primary care patients. Gen Hosp Psychiatry 2003;25:311-5.

43 Gamble VN. Under the shadow of tuskegee: African Americans and health care. Am J Public Health 1997:87:1773-8.

44 Corbie-Smith G, Research AKRJ. Research and Ethics: A Legacy of Distrust. 2nd Edition. San Francisco: Josey-Bass Publishers, 2001.

45 Brotman S, Ryan B, Cormier R. The health and social service needs of gay and lesbian elders and their families in Canada. Gerontologist 2003;43:192-202.

46 Rozbroj T, Lyons A, Pitts M, et al. Assessing the applicability of e-therapies for depression, anxiety, and other mood disorders among lesbians and gay men: analysis of 24 web- and mobile phone-based self-help interventions. J Med Internet Res 2014;16:e166.

47 Pachankis JE, Hatzenbuehler ML, Rendina HJ, et al. LGB-affirmative cognitive-behavioral therapy for young adult gay and bisexual men: a randomized controlled trial of a transdiagnostic minority stress approach. J Consult Clin Psychol 2015;83:875-89.

48 Miranda J, Azocar F, Organista KC, et al. Treatment of depression among impoverished primary care patients from ethnic minority groups. Psychiatr Serv 2003;54:219-25.

49 Miranda J, Chung JY, Green BL, et al. Treating depression in predominantly low-income young minority women: a randomized controlled trial. JAMA 2003;290:57-65.

50 Miranda J, Green BL, Krupnick JL, et al. One-Year outcomes of a randomized clinical trial treating depression in low-income minority women. J Consult Clin Psychol 2006;74:99-111.

51 Arevian AC, Jones F, Tang L, et al. Depression remission from community coalitions versus individual program support for services: findings from community partners in care, Los Angeles, California, 2010-2016. Am J Public Health 2019;109:S205-13.

52 Wells KB, Tang L, Miranda J, et al. The effects of quality improvement for depression in primary care at nine years: results from a randomized, controlled group-level trial. Health Serv Res 2008;43:1952-74.

53 Jones L, Wells K. Strategies for academic and clinician engagement in community-participatory partnered research. JAMA 2007;297:407-10.

54 Jones L, Wells K, Norris K, et al. The vision, Valley, and victory of community engagement. Ethn Dis 2009;19.

55 Wells K, Jones L. "Research" in community-partnered, participatory research. JAMA 2009;302:320-1.

56 Springgate B, Jones C, Allen C, et al. A community-based participatory assessment of health care needs in post-Katrina new Orleans 2007

57 Miranda J, Nakamura R, Bernal G. Including ethnic minorities in mental health intervention research: a practical approach to a longstanding problem. Cult Med Psychiatry 2003;27:467-86.

58 Meyers D, Allen III CE, Dunn D, et al. Community perspectives on post-Katrina mental health recovery in New Orleans. Ethn Dis 2011;21.

59 Wennerstrom A, Vannoy SD, Allen C. Community-Based participatory development of a community health worker mental health outreach role to extend collaborative care in post-Katrina new Orleans. Ethn Dis $2011 ; 21$.

60 Chung B, Jones L, Dixon EL, et al. Using a community partnered participatory research approach to implement a randomized controlled trial: planning community partners in care. $J$ Health Care Poor Underserved 2010;21:780-95.

61 Springgate BF, Wennerstrom A, Meyers D, et al. Building community resilience through mental health infrastructure and training in postKatrina new Orleans. Ethn Dis 2011;21.

62 Arevian AC, O'Hora J, Jones F, et al. Participatory technology development to enhance community resilience. Ethn Dis 2018:28:493-502.

63 Kroenke K, Strine TW, Spitzer RL, et al. The PHQ-8 as a measure of current depression in the general population. $J$ Affect Disord 2009;114:163-73. 
64 Ware J, Kosinski M, Keller SD. A 12-Item short-form health survey: construction of scales and preliminary tests of reliability and validity. Med Care 1996;34:220-33.

65 Ware JE, Kosinski M, Turner-Bowker DM, et al. How to score version 2 of the SF-12 health survey (with a supplement documenting version 1). QualityMetric Incorporated 2002.

66 Ware JE, Sherbourne CD. The mos 36-item short-form health survey (SF-36). I. conceptual framework and item selection. Med Care 1992;30:473-83.

67 Berwick DM, Murphy JM, Goldman PA, et al. Performance of a fiveitem mental health screening test. Med Care 1991;29:169-76.

68 Gibbons RD, Weiss DJ, Pilkonis PA, et al. Development of the CATANX: a computerized adaptive test for anxiety. Am J Psychiatry 2014;171:187-94.

69 Schoenbaum M, Unützer J, Sherbourne C, et al. Cost-Effectiveness of practice-initiated quality improvement for depression. JAMA 2001;286:1325-30.

70 Centers for Disease Control and Prevention. National HIV Behaviora Surveillance (NHBS) MSM Survey [Measurement instrument]. 2016.

71 Wilson EC, Garofalo R, Harris RD, et al. Adolescent medicine trials network for HIV/AIDS interventions. (2009). transgender female youth and sex work: HIV risk and a comparison of life factors related to engagement in sex work. AIDS and Behavior 2009;13:902-13.

72 Weathers FW, Litz BT, Keane TM, et al. The PTSD checklist for DSM5 (PCL-5). National center for PTSD 2013.

73 Bush K, Kivlahan DR, McDonell MB, et al. The audit alcohol consumption questions (AUDIT-C): an effective brief screening test for problem drinking. ambulatory care quality improvement project (ACQUIP). alcohol use disorders identification test. Arch Intern Med 1998;158:1789-95.

74 Bohn MJ, Babor T, Kranzler HR. Validity of the drug abuse screening test (DAST-10) in inpatient substance abusers. Problems of Drug Dependence 1991;119:233-5.

75 Wagnild GM, Young HM. Development and psychometric evaluation of the resilience scale. Journal of Nursing Measurement 1993.

76 Sherbourne CD, Edelen MO, Zhou A, et al. How a therapy-based quality improvement intervention for depression affected life events and psychological well-being over time: a 9-year longitudinal analysis. Med Care 2008;46.

77 Williams DR, Yu Y, Jackson JS, et al. "Racial Differences in Physical and Mental Health: Socioeconomic Status, Stress, and Discrimination.". Journal of Health Psychology 1997;2:335-51.
78 Herek GM, Gillis JR, Cogan JC. Internalized stigma among sexual minority adults: insights from a social psychological perspective. Journal of Counseling Psychology 2009;56:32

79 Lunn MR, Lubensky M, Hunt C, et al. A digital health research platform for community engagement, recruitment, and retention of sexual and gender minority adults in a national longitudinal cohort study - the pride study. Journal of the American Medical Informatics Association, 00(0) 2019:1-12.

80 Miranda J, Storms M. Psychological adjustment of lesbians and gay men. Journal of Counseling \& Development 1989;68:41-5.

81 Littell RC, Milliken G, Stroup W, et al. Sas system for mixed models. Cary, NC: SAS Institute Inc, 1996.

82 Little RJA. Missing-data adjustments in large surveys. Journal of Business \& Economic Statistics 1988;6:287-96.

83 Rubin DB. Inference and missing data. Biometrika 1976;63:581-92.

84 Diggle P, Kenward MG. Informative drop-out in longitudinal data analysis. Applied Statistics 1994;43:49-93.

85 Little RJA. Pattern-mixture models for multivariate incomplete data Journal of the American Statistical Association 1993;88:125-34.

86 Hedeker D, Gibbons RD. Application of random-effects patternmixture models for missing data in longitudinal studies. Psychol Methods 1997;2:64-78.

87 Rosenbaum P. Observational studies. New York: Spinger-Verlag, 1995.

88 Rubin D. Multiple imputation for nonresponse in surveys. Hoboken, NJ: John Wiley \& Sons, 1987.

89 Little RJA, Rubin DB. Statistical analysis with missing data. vol 4. New York: Wiley, 1987

90 McCaffrey DF, Duan N, Morton SC. Propagation of nonresponse weights for censoring in multi-phase screening in complex sample designs. Health Services and Outcomes Research Methodology 2000;1:213-31.

91 Lavori PW, Dawson R, Shera D. A multiple imputation strategy for clinical trials with truncation of patient data. Stat Med 1995;14:1913-25.

92 Korn EL, Graubard BI. Analysis of health surveys. Hoboken, New Jersey: Wiley-Interscience, 1999.

93 Tisnado DM, Adams JL, Liu H, et al. What is the concordance between the medical record and patient self-report as data sources for ambulatory care? Med Care 2006;44:132-40.

94 Rozario PA, Morrow-Howell N, Proctor E. Comparing the Congruency of self-report and provider records of depressed elders' service use by provider type. Med Care 2004;42:952-9. 